\title{
INVERTEBRADOS CAVERNÍCOLAS DA PORÇÃO MERIDIONAL DA PROVÍNCIA ESPELEOLOGICA DO VALE DO RIBEIRA, SUL DO BRASIL
}

\author{
Ricardo Pinto-da-Rocha ${ }^{1}$
}

\begin{abstract}
CAVERNICOLOUS INVERTEBRATE FROM SOUTH SPELEOLOGICAL PROVINCE OF THE RIBEIRA VALLEY, SOUTH BRAZIL. From 1986 to 1992, I surveyed the invertebrate fauna of twelve limestone caves from South Ribeira Valley (State of Paraná, Brazil). Associated to walls in the entrace zone live Enoploctenus and Blechroscelis spiders, Goniosoma opilionids, Strinatia crickets, Latebraria amphipyroides moths, and Culicidae and Sciaridae dipterans. In aphotic zone, occur Plato spiders, Daguerreia inermis opilionids, Pseudonannolene strinatii diplopods, Smicridea caddisflies and the aquatic crustacean Aegla paulensis. I found in both zones Ctenus fasciatus spiders, Paronella collembolans, Zelurus travassosi heteropterans, Cholevidae and Anthribidae beetles, Phoridae dipterans and Braconidae wasps. One of the most important energy sources in these caves is the hematophagous bat guano. Associated to the guano piles there is a fauna composed by psedoscorpiones, acarians, Katantodesmus diplopods, Acherontides and Paronella collembolans, Dissochaetus beetles and Phoridae dipterans. The cave fauna studied is similar to that Ribeira Valley of the State of São Paulo. The most expressive difference is the absence of some taxa very common in São Paulo caves, such as the Oniscidae and Styloniscidae isopods, Cryptodesmidae and Chelodesmidae diplopods, Endecous crickets, Tricommatidae harvestmen, Calamoceratidae caddisflies and Neoditomyia Keroplatidae dipterans.

KEY WORDS, Cave fauna, distribution, guano, South Brazil, Troglomorphic taxa
\end{abstract}

$\mathrm{O}$ ambiente cavernícola caracteriza-se por possuir, nas zonas mais afastadas dos contatos com o meio externo, condições climáticas praticamente estáveis, com umidade relativa alta e tendendo à saturação, temperatura com pequena variação circadiana e circanual, e a mais notável, ausência absoluta de luz (BARR \& HOLSINGER, 1985). Esse meio é pobre em matéria orgânica, sendo suas fontes inconstantes e importadas do exterior, através de entrada por cursos d'água, por fendas e outras comunicações e por morcegos (CULVER, 1982).

As cavernas são colonizadas por invertebrados epígeos que habitam, no caso da fauna terrestre, principalmente o solo e folhiço da vegetação externa (CULVER, 1982), como por exemplo, Acari, Collembola e Diplopoda. Uma

1) Museu de Zoologia da Universidade de São Paulo, Caixa Postal 7172, 01064-970 São Paulo, São Paulo, Brasil. 
vez isolados no meio subterrâneo, esses organismos podem especializar-se a tal ponto que não mais sobrevivem no meio externo, tornando-se o que se denomina troglóbio.

A composição da fauna cavernícola do Brasil é relativamente bem conhecida, estando estas informações disponíveis em CHAIMOWICZ (1984; 1986); DESSEN et al. (1980); GNASPINI-NETTO (1989); GODOY (1986) e principalmente TRAJANO $(1985 ; 1987)$ e TRAJANO \& GNASPINI-NETTO (1991a). Porém, pouco se conhece sobre as grutas do Estado do Paraná, sendo que nunca foi feito um levantamento bioespeleológico sistemático. A maioria dos trabalhos publicados, que incluíam informações sobre animais cavernícolas paranaenses, tratam-se de descrição ou registro de táxons, como em CARVALHO (1989), EICKSTEDT (1975), GNASPINI (1991), GNASPINI-NETTO (1989), NOGUEIRA (1959), SOARES \& SOARES (1947), TRAJANO $(1985 ; 1987)$.

O presente estudo, publicado preliminarmente em PINTO-DAROCHA (1989), tem por objetivo fornecer uma visão sobre as comunidades subterrâneas do Paraná, bem como contribuir para o conhecimento da fauna cavernícola brasileira.

\section{MATERIAL E MÉTODOS}

\section{Descrição da área.}

As 12 cavernas estudadas situam-se em rochas carbonáticas (calcário e dolomito) do grupo Açungui (Proterozóico Superior), na porção paranaense da Província Espeleológica do Vale do Ribeira, sul do Brasil (sensu KARMANN \& SÁNCHEZ, 1986). Segundo a classificação de AB'SABER (1977), a área de estudo situa-se na transição do Domínio dos Planaltos de Araucárias com o Domínio Atlântico. O clima é quente-temperado, sempre úmido, do tipo $\mathrm{Cfb}$ segundo a classificação de Koeppen, apresentando médias mensais de temperatura entre 11,7 e $20,1^{\circ} \mathrm{C}$ e precipitação anual próxima a $1470 \mathrm{~mm}$ (MAACK, 1981).

A porção paranaense do Vale do Ribeira vem sofrendo uma intensa atividade antrópica, que está descaracterizando o ambiente, onde, em diversos locais, a vegetação original foi totalmente retirada e substituída por plantações de bracatinga (Mimosa scabrella, Leguminosae) e por culturas de subsistência como a do milho e do feijão (KLEIN, 1962). As Grutas do Bacaetava, Jesuítas, Lancinha, Olhos d'Água e Pinheiro Seco recebem uma visitação humana regular, que causa sérios danos ao ecossistema como: compactação do solo; pisoteamento de animais e aumento da matéria orgânica disponível na forma de fezes e restos de alimentos.

Segue-se a lista das cavernas amostradas, com informações relativas ao: número de visitas realizadas pelo autor entre 1986 e 1990 (entre parênteses), características topográficas e fontes alimentares conspícuas e disponíveis aos cavernícolas. Os dados sobre localização, rocha encaixante, desenvolvimento, 
presença de curso d'água, encontram-se na tabela I e foram extraídos de SOCIEDADE BRASILEIRA DE ESPELEOLOGIA (1989).

Tabela I. Dados sobre as cavernas amostradas. Rocha $(\mathrm{C}=$ calcário, $\mathrm{D}=$ dolomita, $\mathrm{M}=$ mármore $)$, número de cadastro na Sociedade Brasileira de Espeleologia, coordenadas geográficas, município, altitude, desenvolvimento e presença de curso d'água. Fonte: SOCIEDADE BRASILEIRA DE ESPELEOLOGIA (1989).

\begin{tabular}{|c|c|c|c|c|c|c|}
\hline Caverna (rocha) & Cadastro & Coordenadas geográficas & Município & Altitude & Desenvolvimento & Rio \\
\hline Água Boa (D) & PR-16 & $25^{\circ} 16^{\prime} 45^{\prime \prime} \mathrm{S}-49^{\circ} 21^{\prime} 30^{\prime \prime} \mathrm{W}$ & Almirante Tamandaré & $980 \mathrm{~m}$ & $80 \mathrm{~m}$ & ausente \\
\hline Bacaetava (D) & PR-03 & $25^{\circ} 14^{\prime} 05^{\prime \prime} \mathrm{S}-49^{\circ} 12^{\prime} 23^{\prime \prime} \mathrm{W}$ & Colombo & $900 \mathrm{~m}$ & $455 \mathrm{~m}$ & presente \\
\hline Bromados I (C) & PR-27 & $25^{\circ} 04^{\prime} 33^{\prime \prime} \mathrm{S}-49^{\circ} 22^{\prime} 48^{\prime \prime} \mathrm{W}$ & Rio Branco do Sul & $800 \mathrm{~m}$ & $300 \mathrm{~m}$ & presente \\
\hline Itacolombo (D) & PR-18 & $25^{\circ} 11^{\prime} 11^{\prime \prime} \mathrm{S}-49^{\circ} 14^{\prime} 46^{\prime \prime} \mathrm{W}$ & Rio Branco do Sul & $990 \mathrm{~m}$ & $500 \mathrm{~m}$ & presente \\
\hline Jesuitas (C) & PR-09 & $25^{\circ} 03^{\prime} 00^{\prime \prime} \mathrm{S}-49^{\circ} 04^{\prime} 20^{\prime \prime} \mathrm{W}$ & Bocaiúva do Sul & $918 \mathrm{~m}$ & $1130 \mathrm{~m}$ & presente \\
\hline Lancinha (D) & PR-06 & $25^{\circ} 19^{\prime} 58^{\prime \prime} \mathrm{S}-49^{\circ} 17^{\prime} 12^{\prime \prime \prime} \mathrm{W}$ & Rio Branco do Sul & $886 \mathrm{~m}$ & $1700 \mathrm{~m}$ & presente \\
\hline Olhos d'Água (D) & PR-50 & $25^{\circ} 01^{\prime} 23^{\prime \prime} \mathrm{S}-49^{\circ} 47^{\prime} 30^{\prime \prime} \mathrm{W}$ & Castro & $750 \mathrm{~m}$ & $500 \mathrm{~m}$ & presente \\
\hline Pinheirinho (M) & PR-23 & $25^{\circ} 00^{\prime} \mathrm{S}-49^{\circ} 38^{\prime} \mathrm{W}$ & Campo Largo & $500 \mathrm{~m}$ & $800 \mathrm{~m}$ & presente \\
\hline Pinheiro Seco (D) & PR-47 & $25^{\circ} 43^{\prime} 40^{\prime \prime} \mathrm{S}-49^{\circ} 32^{\prime} 50^{\prime \prime} \mathrm{W}$ & Castro & $800 \mathrm{~m}$ & $650 \mathrm{~m}$ & ausente \\
\hline Terra Boa (C) & PR-15 & $25^{\circ} 12^{\prime} 58^{\prime \prime} \mathrm{S}-49^{\circ} 31^{\prime} 23^{\prime \prime} \mathrm{W}$ & Almirante Tamandaré & $667 \mathrm{~m}$ & $1000 \mathrm{~m}$ & presente \\
\hline Toca (C) & PR-14 & $25^{\circ} 10^{\prime} 38^{\prime \prime} \mathrm{S}-49^{\circ} 18^{\prime} 16^{\prime \prime} \mathrm{W}$ & Rio Branco do Sul & $900 \mathrm{~m}$ & $78 \mathrm{~m}$ & ausente \\
\hline Toquinhas (D) & PR-20 & $25^{\circ} 09^{\prime} 56^{\prime \prime} \mathrm{S}-49^{\circ} 18^{\prime} 05^{\prime \prime} \mathrm{W}$ & Rio Branco do Sul & $910 \mathrm{~m}$ & $260 \mathrm{~m}$ & presente \\
\hline
\end{tabular}

* Gruta da Água Boa (quatro visitas) possui duas entradas e uma galeria em forma de "V". Fontes alimentares: guano de morcegos hematófagos em quase toda gruta.

* Gruta do Bacaetava (3) é composta por uma galeria superior seca de $115 \mathrm{~m}$ e uma inferior, com rio, de $455 \mathrm{~m}$. A superior apresenta uma entrada de pequenas dimensões, e a inferior, duas entradas de grandes dimensões. Detritos vegetais carregados pelo rio, pequenos acúmulos de guano $\left(150 \mathrm{~cm}^{2}\right)$ de hematófagos, frugívoros e carnívoros.

* Gruta de Bromados (3) possui três entradas, duas delas de pequenas dimensôes, comunicando-se com a galeria principal, que apresenta pequenos salões. Detritos vegetais carreados através das comunicações com o exterior e acúmulos esparsos de guano de hematófagos.

* Gruta de Itacolombo (3) possui uma entrada impenetrável, de pequenas dimensões, próxima da ressurgência. Outras duas entradas e um salão de grandes dimensões comunicam-se com a galeria principal, que é percorrida em toda a sua extensão por rio. Também conhecida como Gruta de Campestrinho. Detritos vegetais trazidos pelo rio e através das entradas, grandes acúmulos de guano de hematófagos (de aproximadamente $2500 \mathrm{~cm}^{2}$ ).

* Gruta dos Jesuítas (4) situada no Parque Estadual de Campinhos, também conhecida como Gruta de Campinhos. Possui como entradas o sumidouro, e duas entradas secas, todas de grandes dimensões. A galeria principal é parcialmente percorrida por rio. Apresenta alguns salōes e várias galerias laterais. Troncos podres que serviam como escadas, pequenos acúmulos de guano de hematófagos e carnívoros. 
* Gruta da Lancinha (23) é a maior gruta do Paraná, recebendo visitação humana principalmente na galeria do rio. Apresenta três entradas de grandes dimensões; o sumidouro, a ressurgência e uma entre estas duas, em local onde houve um desabamento. Possui uma galeria percorrida por rio, com vários salōes superiores de pequenas dimensões, e outra seca, com salōes de grandes dimensões. Detritos vegetais trazidos pelo rio e através das comunicações com o exterior, fezes de lontra, raízes de plantas, pequenos e grandes acúmulos de guano de hematófagos e carnívoros.

* Gruta Olhos d'Água (2) apresenta uma pequena entrada próxima ao sumidouro, uma galeria percorrida por rio, com duas clarabóias, e outra entrada no final desta galeria. Detritos vegetais trazidos pelo rio e através de comunicações com o exterior e pequenos acúmulos de guano de hematófagos e carnívoros.

* Gruta de Pinheirinho (2) possui uma entrada de pequenas dimensões e uma galeria com pequenos salóes, percorrida por rio. Fezes de lontra e pequenos acúmulos de guano de hematófagos.

* Gruta de Pinheiro Seco (1) possui duas entradas e uma galeria (parcialmente dinamitada). Pequenos acúmulos de guano de hematófagos.

* Gruta de Terra Boa (3) possui uma entrada de pequenas dimensões, seguida por galeria, percorrida por rio na maior parte do seu desenvolvimento. Detritos vegetais trazidos pelo rio, raízes de plantas, pequenos acúmulos de guano de hematófagos, carnívoros e insetívoros.

* Gruta da Toca (6) apresenta duas entradas de pequenas dimensões e uma galeria com desnível de $17 \mathrm{~m}$. Detritos vegetais.

*Gruta de Toquinhas (8) apresenta uma entrada próxima ao sumidouro impenetrável e outra a $60 \mathrm{~m}$ deste; possui uma galeria percorrida por rio e outras duas secas, paralelas a esta. Detritos vegetais trazidos pelo rio e através de comunicações com o exterior, raízes de plantas e pequenos acúmulos de guano de hematófagos e carnívoros.

\section{Trabalho de campo e de laboratório.}

As coletas e observações foram realizadas nas 12 cavernas, em diferentes épocas do ano, de fevereiro de 1986 até março de 1992.

Os invertebrados terrestres foram coletados utilizando-se as seguintes técnicas: coleta manual, com pinça e pincel; coleta de solo, guano e folhiço, com macrotriagem no local e triagem em funil de Tulgreen-Berlese em laboratório; armadilhas de formalina sem ou com isca de fígado de galinha, enterradas no sedimento por vários dias; rede entomológica. Os crustáceos aquáticos foram capturados com covos iscados com fígado de galinha. Algumas amostras de guano foram mantidas em laboratório para a obtenção de insetos adultos. $\mathrm{O}$ guano de hematófagos, citado neste trabalho, é produzido pelo morcego Desmodus rotundus (Phyllostomidae), e o de carnívoros é produzido pelo Chrotopterus auritus (Phyllostomidae) e a(s) espécie(s) do guano de insetívoros não foi determinada. Estas técnicas visaram obter uma maior abrangência 
qualitativa de fauna cavernícola, porém nem todas foram aplicadas em cada caverna.

O material obtido encontra-se depositado nas seguintes instituições: Museu Nacional do Rio de Janeiro, Instituto Butantan, Departamento de Zoologia do Instituto de Biociências da Universidade de São Paulo, Departamento de Parasitologia do Instituto de Ciências Biomédicas da Universidade de São Paulo, Museu de Zoologia da Universidade de São Paulo, Universidade Estadual Paulista "Julio Mesquita Filho" campus Rio Claro, Museu de História Natural "Capão da Imbuia", Departamento de Zoologia da Universidade Federal do Paraná e Museu de Ciências Naturais da Fundação Zoobotânica do Rio Grande do Sul.

\section{RESULTADOS}

Apresenta-se a seguir, a lista dos táxons e cavernas onde foram registrados, com observações sobre sua distribuição geográfica, hábitats preferenciais, recursos alimentares utilizados e outras observações de cunho ecológico, além da presença de troglomorfismos (regressão ocular e/ou pigmentar). Os cavernícolas foram classificados em: troglóbio, que estão restritos ao ambiente subterrâneo e são incapazes de sobreviver fora dele e podem apresentar troglomorfismos; troglófilos, que são capazes de realizar todo o ciclo de vida tanto fora quanto no meio hipógeo; e trogloxenos, que dependem, pelo menos em parte do meio epígeo.

\section{Filo ANNELIDA}

\section{Classe OLIGOCHAETA}

Ocorrência: Água Boa, Bacaetava, Jesuítas, Lancinha, Olhos d'Água, Pinheiro Seco, Toca, Toquinhas

Observações: Os oligoguetos foram encontrados em bancos de sedimentos, madeiras em decomposição e guano de morcegos hematófagos.

\section{Filo ARTHROPODA}

Classe ARACHNIDA

\section{Ordem PSEUDOSCORPIONES}

Chernetidae, Hesperochernetinae, V. Mahnert det.

Ocorrência: Água Boa, Bacaetava, Itacolombo, Jesuítas, Lancinha Olhos d'Água, Pinheirinho, Terra Boa, Toca, Toquinhas.

\section{Ideoroncidae}

Ideoroncus sp., V. Mahnert det.

Ocorrência: Lancinha

Observações: As duas espécies de pseudoescorpiões não apresentam 
troglomorfismos como despigmentação ou hipertrofia dos apêndices (V. Mahnert in litteris). Chernetidae é comum em cavernas do Vale da Ribeira e em outras regiões do Brasil, e nas cavernas estudadas deve ser troglófila. Já a espécie de Ideoroncus foi coletada uma única vez e sobre sua classificação ecológica nada se pode afirmar. Estes aracnídeos são predadores, que habitam principalmente o folhiço e guano de morcegos, desde a zona de entrada até mais distantes, e alimentam-se de larvas de dípteros (e.g. Phoridae) e de coleópteros (e.g. Dissochaetus), colêmbolos e ácaros (TRAJANO \& GNASPINI-NETTO, 1991).

\section{Ordem ARANEAE}

\section{Loxoscelidae}

Loxosceles gaucho Gertsch, 1967, E. Buckup det.

Ocorrência: Bromados, Itacolombo, Olhos d'Água, Toquinhas

\section{Pholcidae}

Blechroscelis sp., R. Baptista det.

Ocorrência: Água Boa, Bacaetava, Itacolombo, Lancinha, Pinheirinho, Pinheiro Seco, Terra Boa, Toca, Toquinhas.

\section{Theridiidae}

Achaearanea sp., R. Baptista det.

Ocorrência: Água Boa, Lancinha, Terra Boa, Toca.

\section{Theridiosomatidae}

Plato sp., Coddington det.

Ocorrência: Bacaetava, Bromados, Jesuítas, Itacolombo, Lancinha, Olhos d'Água, Pinheirinho, Pinheiro Seco, Terra Boa, Toca, Toquinhas.

\section{Pisauridae}

Trechalea keyserlingi F.Cambridge, 1903, R.Baptista det.

Ocorrência: Água Boa, Bacaetava, Jesuítas, Lancinha, Olhos d'Água, Pinheirinho, Pinheiro Seco, Terra Boa.

\section{Ctenidae}

Ctenus fasciatus Mello-Leitão, 1943, E.Buckup det.

Ocorrência: Água Boa, Bromados, Itacolombo, Lancinha, Olhos d'Água, Pinheiro Seco, Terra Boa, Toca, Toquinhas.

Enoploctenus sp., E. Buckup det.

Ocorrência: Bromados, Lancinha, Olhos d'Água, Pinheirinho, Pinheiro Seco, Toca.

\section{Lyniphiidae, Erigoniinae}

Neomaso sp., R. Baptista det.

Ocorrência: Lancinha

Observações: A fauna de aranhas é similar à de São Paulo, sendo composta por pelo menos oito espécies de trogloxenos e troglófilos. A aranhas Theridiosomatidae vivem quase que exclusivamente em ambientes úmidos e escuros e quando em cavernas constroem teias nas paredes (CODDINGTON, 
1986). Representantes do gênero Plato são muito comuns, com registros em 11 das 12 cavernas estudadas; são troglófilos, alimentam-se de pequenos dípteros (e.g. Chironomidae e Phoridae) e tricópteros e são mais abundantes próximos a cursos d'água. Outra espécie troglófila amplamente distribuída pelas cavernas do Paraná é Ctenus fasciatus, uma aranha errante, encontrada em toda a extensão das cavernas. Esta aranha ocorre na maioria das grutas do Vale do Ribeira (EICKSTEDT, 1975; TRAJANO, 1987), alimentando-se de grilos, opiliões e talvez diplópodos (TRAJANO \& GNASPINI-NETTO, 1991b). Outras espécies cavernícolas do gênero Ctenus são encontradas nas Províncias Espeleológicas do Bambuí, Serra da Bodoquena e Serra Geral (TRAJANO \& GNASPINI-NETTO, 1991a). Enoploctenus sp. é um trogloxeno registrado na zona de entrada em seis cavernas, observando-se sempre um ou dois indivíduos. A aranha-marrom, Loxosceles spp., é sedentária, construindo teias irregulares, e alimenta-se de dípteros (EICKSTEDT, 1975), coleópteros e diplópodes (TRAJANO \& GNASPINI-NETTO, 1991b). No Paraná, L. guacho foi registrada em apenas quatro cavernas, sempre próxima a comunicações com o exterior, situação diversa da observada em São Paulo, onde populações de Loxosceles ocorrem na maioria das cavernas, sem se restringirem à zona de entrada, sendo consideradas troglófilas (TRAJANO, 1987). Achaearanea sp. também foi registrada em quatro grutas, sendo que na Gr. da Lancinha construiu teia com fios presos nas parede e na lâmina d'água. Outras aranhas trogloxenas comuns na zona de entrada são Trechalea keyserlingi, espécie errante que caça por sobre a água, e Blechroscelis sp. que constrói teia nas paredes, sempre na região de entrada. Neomaso sp. foi registrada, uma única vez, coletada em teia no guano de hematófagos.

\section{Ordem OPILIONES}

\section{Gonyleptidae}

Goniosominae

Goniosoma aff. badium C.L. Koch, 1839, R. Pinto-da-Rocha det.

Ocorrência: Água Boa, Bromados, Itacolombo, Jesuítas, Lancinha, Olhos d'Água, Pinheirinho, Terra Boa, Toca, Toquinhas.

Mitobatinae

Promitobates hatschbachi H. Soares, 1945, R. Pinto-da-Rocha det.

Ocorrência: Água Boa, Pinheirinho

Pachylinae

Daguerreia inermis Soares \& Soares, 1947, Pinto-da-Rocha det.

Ocorrência: Água Boa, Bacaetava, Itacolombo, Jesuítas, Lancinha, Olhos d'Água, Pinheirinho.

Observações: Os opiliões são representados por três espécies de Gonyleptidae, duas amplamente distribuídas pelas cavernas estudadas, e outra ( $P$. hatschbachi) observada em apenas duas ocasiões, podendo ser trogloxena. Goniosoma aff. badium é um trogloxeno característico das paredes na zona de 
entrada, onde foram observadas agressões de nove a 34 exemplares e também posturas de 72 a 105 ovos, próximos uns dos outros e com uma fêmea sempre próxima. Jovens e adultos de Daguerreia inermis, troglófilo cuja localidade-tipo é a Gr. dos Jesuítas, são encontrados em todas as zonas, em muitas cavernas carbonáticas do Vale do Ribeira. Ambas são onívoras, predando invertebrados e alimentando-se de detritos vegetais e animais mortos. Na Gr. de Olhos d'Água foi observado G.aff. badium predando tricópteros. Os principais predadores destes opiliões são o heteróptero Zelurus travassosi e aranha Ctenus fasciatus.

\author{
Sub-Classe ACARI \\ Ordem GAMASIDA
}

\title{
Parasitidae
}

Eugamasus butleri Hughes, 1948 D. Baggio det.

Ocorrência: Olhos d'Água

Parasitus sp., D. Baggio det.

Ocorrência: Itacolombo

gênero indet., D. Baggio det.

Ocorrência: Pinheirinho, Toquinhas

\section{Macrochelidae}

Holocelaeno sp., D. Baggio det.

Ocorrência: Olhos d'Água

Macrocheles muscaedomesticae (Scopoli, 1772), D. Baggio det. nhas.

Ocorrência: Água Boa, Itacolombo, Olhos d'Água, Terra Boa, Toqui-

\section{Gamasidae}

Hologamasida sp., D. Baggio det.

Ocorrência: Itacolombo

\section{Phytoseiidae}

Neoseiulus barkeri Hughes, 1948, D. Baggio det.

Ocorrência: Jesuítas

\section{Ascidae}

Blattissocius tarsalis (Berlese, 1918) D. Baggio det. Registro duvidoso.

Ocorrência: Água Boa, Bacaetava, Itacolombo, Terra Boa, Toquinhas.

Melichares agilis Hering, 1838 D. Baggio det.

Ocorrência: Olhos d'Água

\section{Laelapidae}

Eulaelaps sp., D. Baggio det.

Ocorrência: Lancinha, Terra Boa

Hypoaspis aculifer Canestrini, 1884, D. Baggio det.

Ocorrência: Jesuítas 
Laelaps nutalli Hirst, 1915, D. Baggio det.

Ocorrência: Bacaetava, Lancinha, Toquinhas

Proctolaelaps sp., D. Baggio det.

Ocorrência: Água Boa, Bacaetava, Bromados, Itacolombo, Lancinha, Pinheirinho, Terra Boa, Toca, Toquinhas

\section{Thinozerconidae}

Thinozercon sp., D. Baggio det.

Ocorrência: Itacolombo

\section{Uropodidae}

Uropoda sp., D. Baggio det.

Ocorrência: Itacolombo

\section{Frediziidae}

Fredizzia sp., D. Baggio det.

Ocorrência: Lancinha

\section{Celaenopsidae}

Celaenopsis kutensis Kimm, 1968, D. Baggio det.

Ocorrência: Itacolombo

\section{Ordem IXODIDA}

\section{Argasidae}

Ornithodoros talaje Guérin \& Menevile, 1849, D. Baggio det.

Ocorência: Lancinha

\section{Ixodidae}

Ixodes sp., D. Baggio det.

Ocorrência: Terra Boa

\section{Ordem ACTINEDIDA}

\section{Bdelidae}

Spinidbella cronini (Baker \& Balock, 1944), D. Baggio det.

Ocorrência: Jesuítas, Terra Boa, Toquinhas

\section{Tydeidae}

Tydeus interruptus Sig Thor, 1932, D. Baggio det.

Ocorrência: Toquinhas

Tydeus sp., D. Baggio det.

Ocorrência: Lancinha

\section{Cheyletidae}

Cheyletus malaccensis Oudemans, 1903, D. Baggio det.

Ocorrência: Itacolombo 


\section{Smaridiidae}

Smarida frustoris Banks, 1940, D. Baggio det.

Ocorrência: Terra Boa

\section{Ordem ASTIGMATA}

\section{Acaridae}

Rhizoglyphus echinopus (Fumouze \& Robin, 1868), D. Baggio det.

Ocorrência: Olhos d'água

Tyrophagus putrescentiae (Schrank, 1781), D. Baggio det.

Registro duvidoso.

Toquinhas

Ocorrência: Água Boa, Bacaetava, Itacolombo, Lancinha, Olhos d'Água,

\section{Glycyphagidae}

Caloglyphus berlesei (Michael, 1903), D. Baggio det.

Ocorrência: Itacolombo, Terra Boa

Glycyphaggus domesticus (DeGeer, 1778), D. Baggio det.

Ocorrência: Pinheirinho

\section{Pyroglyphidae}

Dermatophagoides sp., D. Baggio det.

Ocorrência: Itacolombo

\section{Ordem ORIBATIDA}

\section{Epilohmanniidae}

Epilohmannia dolosa Perez-Inigo \& Baggio, 1985, D. Baggio det. Ocorrência: Pinheirinho

\section{Phthiracaridae}

Steganacarus fonseciai Perez-Inigo \& Baggio, 1980, D. Baggio det.

Ocorrência: Jesuítas, Terra Boa, Toquinhas

\section{Cepheidae}

Tectocepheus americanus Perez-Inigo \& Baggio, 1989, D. Baggio det.

Ocorrência: Pinheirinho

\section{Oppiidae}

Brachioppia tropicalis Perez-Inigo \& Baggio, 1980, D. Baggio det.

Ocorrência: Jesuítas

Quadrioppia sp., D. Baggio det.

Ocorrência: Terra Boa

\section{Galumnidae}

Galumna inexa Perez-Inigo \& Baggio, 1986, D. Baggio det.

Ocorrência: Lancinha, Pinheirinho 
Pergalumna nasica Perez-Inigo \& Baggio, 1980, D. Baggio det.

Ocorrência: Itacolombo, Pinheirinho, Toquinhas

\section{Oribatulidae}

Peloribates anomalus Perez-Inigo \& Baggio, 1980, D. Baggio det.

Ocorrência: Jesuítas, Lancinha

Observações: Os ácaros constituem o grupo com maior número de espécies nas grutas estudadas. Foram registrados 38 táxons de ácaros, pertencendo 17 a Gamasida, dois a Ixodida, cinco a Actinedida, cinco a Astigmata e oito a Oribatida. Foram encontrados em bancos de sedimento (ao lado do rio), no solo (distante do rio), em folhiço, fezes de lontra, guano de carnívoros e de hematófagos.

Os membros das famílias Parasitidae, Macrochelidae, Phytoseiidae, Ascidae e Laelapidae são normalmente predadores de microartópodos (KRANTZ, 1975). Dentre estes, destacam-se pela distribuição e abundância os seguintes: Macrocheles muscaedomesticae, Blattissocius tarsalis e Proctolaelaps sp. M. muscaedomesticae utiliza dípteros como meio de dispersão (foresia), efetuando postura de ovos em fezes de aves e mamíferos, onde se alimenta de ovos e larvas de dípteros (FLETCHMANN, 1977). Um indivíduo desta espécie foi registrado em fezes de lontra e populações bem maiores em guano de carnívoros e hematófagos. B. tarsalis é um predador de ácaros e de larvas de coleópteros e foi encontrado em banco de sedimento, fezes de lontra e guano de hematófagos, sempre um exemplar em cada local. Proctolaelaps sp. encontra-se amplamente distribuídos nas grutas estudadas e ocorre em banco de sedimento, solo, folhiço, fezes de lontra, guano de hematófagos. As espécies de Thinozerconidae e Uropodidae foram encontradas em guano de insetívoros. Fredizzia sp. pode ser predadora ou micófaga (KRANTZ, 1975). Os ácaros Ixodida são ectoparasitas de morcegos e tanto O. talaje, como Ixodes sp. foram encontrados no solo.

Os ácaros Actinedida das famílias Bdelidae e Cheyletidae são predadores de ácaros (FLETCHMANN, 1983). As espécies de Tydeus podem ser micófagas (FLETCHMANN, 1983), predadoras ou sugadoras de plantas (KRANTZ, 1975).

A ordem Astigmata apresenta cinco espécies das famílias Acaridae, Glycyphagidae e Phyroglyphidae. Tyrophagus putrescetiae foi registrado em seis cavernas em solo, folhiço, banco de sedimento, guano de carnívoros e hematófagos. Segundo FLETCHMANN (1983) esta espécie alimenta-se de fungos. Espécies de Caloglyphus podem ser saprófagas (KRANTZ, 1975) ou predadores de ovos e larvas de insetos (FLETCHMANN, 1983), C. berlesei foi encontrado no solo, em fezes de lontra e guano de hematófagos.

A ordem Oribatida está amplamente representada nas cavernas do Paraná, estes ácaros normalmente habitam a camada de solo mais rica em matéria orgânica, alimentando-se de detritos vegetais ou de fezes (KRANTZ, 1975). Steganacartus fonseciai, Tectocepheus americanus e Pergalumna nasica foram encontrados em folhiço. Epilohmannia dolosa, Galumna inexa e 
Peloribates anomalus ocorreram em guano de hematófagos e Quadrioppia sp. e Galumna inexa no solo.

Os ácaros Tryrophagus putrescentiae e Blatissocius tarsalis foram encontrados após extração em funil de Tulgreen-Berlese em amostras de seis e cinco cavernas, respectivamente. Porém, seu registro para as cavernas do Paraná é duvidoso, pois eles foram encontrados em grandes quantidades (10 ácaros em cada 0,001 grama de poeira) na poeira do chão e dos móveis do laboratório onde estavam os funis, e seu registro é raro fora do ambiente domiciliar (D. Baggio com. pes.).

\section{Classe CRUSTACEA}

\section{Ordem ISOPODA}

Philosciidae, L.A. Souza det.

Ocorrência: Lancinha, Olhos d'Água

\section{Platyarthridae}

Trichorhina sp., A.L. Castro det.

Ocorrência: Lancinha, Olhos d'Água

Observações: Os isópodos terrestres (Oniscoidea) foram encontrados, em duas ocasiões, em detritos vegetais próximos a entradas. Os exemplares de Trichorhina apresentam despigmentação, porém esta característica também é encontrada em populações epígeas (A.L. Castro, com. pes.), foram registrados sempre em detritos vegetais proximos a comunicações com o meio externo.

\section{Ordem DECAPODA}

\section{Aeglidae}

Aegla paulensis ,G. Bond-Buckup det.

Ocorrência: Bacaetava, Lancinha

Observações: Os exemplares de $A$. paulensis foram encontrados em toda a extensão do rio na Gr. do Bacaetava e Gr. da Lancinha. Representantes deste gênero são comumente encontrados em cavernas de São Paulo (TRAJANO, 1987), alimentam-se de detritos vegetais e animais mortos (TRAJANO \& GNASPINI-NETTO, 1991a).

\section{Classe CHILOPODA}

\section{Ordem LITHOBIOMORPHA}

Lithobiidae, L. Fisman det.

Ocorrência: Lancinha 


\section{Ordem SCOLOPENDROMORPHA}

Cryptopidae, L. Fismam det.

Ocorrência: Toca

Observaçôes: Um único exemplar de Chilopoda foi coligido em cada uma das duas cavernas, na zona de entrada, o que indica serem acidentais nessas cavernas.

\section{Classe DIPLOPODA}

\section{Ordem PSEUDONANNOLENIDA}

\section{Pseudonannolenidae}

Pseudonannolene strinatii Mauriés, 1974, C.S. Fontanetti det.

Ocorrência: Itacolombo, Lancinha, Pinheirinho, Terra Boa, Toca, Toquinhas

\section{Ordem POLYDESMIDA}

\section{Oniscodesmidade}

Katantodesmus sp., R.L. Hoffman det.

Ocorrência: Lancinha, Olhos d'Água, Pinheirinho, Terra Boa Strongylosomidae, R.L. Hoffman det.

Ocorrência: Bromados, Jesuítas

Observações: Os diplópodos que colonizaram efetivamente as cavernas brasileiras pertencem às ordens Pseudonannolenida e Polydesmida, esta última com espécies troglomórficas (TRAJANO, 1987). Populações troglófilas de Pseudonannolene são encontradas em cavernas na Prov. Esp. do Vale do Ribeira e do Bambuí. Em quase todas as cavernas estudadas foram encontradas $P$. strinatii. Espécimes deste gênero são detritívoros errantes e foram observados, nas zonas de entrada e afótica, nas paredes e no solo, folhiço e guano de hematófagos. Várias dezenas de indivíduos de Katantodesmus foram observados, em diversas ocasióes, em guano de hematófagos, tanto em fezes esparsas como em grandes acúmulos de guano. Foram encontrados dois indivíduos em folhiço na Gruta de Olhos d'Água. Estes diplópodos apresentam despigmentação cutânea, situação não observada em outras espécies paranaenses, epigeas, deste gênero descritas por SCHUBART (1953). Esta característica parece ser um troglomorfismo em Katantodesmus.

\section{Classe HEXAPODA}

\section{Ordem COLLEMBOLA}

\section{Hypogastruridae}

Acherontides sp., P. Gnaspini-Netto det.

Ocorrência: Água Boa, Jesuítas, Lancinha, Olhos d'Água, Pinheirinho, Terra Boa 


\section{Neanuridae}

Brachystomella sp., E.P. Oliveira det.

Ocorrência: Toquinhas

\section{Isotomidae}

Isotomiella sp., E.P. Oliveira det.

Ocorrência: Água Boa, Itacolombo, Jesuítas, Lancinha, Olhos d'Água

\section{Entomobryidae}

Lepidocyrtus sp., E.P. Oliveira det.

Ocorrência: Olhos d'Água

Pseudosinella sp., E.P. Oliveira det.

Oocorrência: Lancinha

\section{Cyphoderidae}

Cyphoderus sp., E.P. Oliveira det.

Ocorrênciạ: Lancinha, Olhos d'Água

Oncopoduridae, P. Gnaspini-Netto det.

Ocorrência: Pinheirinho

\section{Paronellidae}

Paronella sp., E.P. Oliveira det.

Ocorrência: Bromados, Itacolombo, Jesuítas, Lancinha, Olhos d'Água, Pinheiro Seco, Terra Boa, Toquinhas

Trogolaphysa sp., E. P. Oliveira det.

Ocorrência: Lancinha

\section{Arrhopalitidae}

Arrhopalitites sp., E.P. Oliveira det.

Ocorrência: Lancinha, Toquinhas

Observações: Os colêmbolos foram encontrados principalmente em guano fresco de hematófagos, onde foram registrados Brachystomella sp., Isotomiella sp., Paronella sp. e destacando-se Acherontides sp. pelas populaçôes muito numerosas em guano fresco de hematófagos. Acherontides parece apresentar troglomorfismo (unha maior que o tibio-tarso), caso isto seja confirmado, deverá ser considerado troglóbio. Isotomiella sp. ocorre em guano de hematófagos, de carnívoros, em folhiço e no solo. Lepidocyrtus sp. e Cyphoderus sp. foram registrados nas grutas paranaenses e ocorrem em guano de hematófagos (um a cinco indivíduos, observados), de carnívoros, fezes de lontra, solo, folhiço e em troncos em decomposição. Arrhopalites sp. foi capturado apenas com armadilha de formalina, na Gr. da Lancinha estava próxima a guano de hematófagos e na Gr. da Toquinhas estava próxima a folhiço. Os colêmbolos Hypogastruridae, Isotomidae e Paronellidae também são comuns nas cavernas de São Paulo (TRAJANO \& GNASPINI-NETTO, 1991a). 


\section{Ordem EPHEMEROPTERA}

\section{Baetidae}

Callibaetis sp., E.R. Silva det.

Ocorrência: Lancinha

Pseudocleon sp., E.P. Silva det.

Ocorrência: Olhos d'Água

\section{Leptophlebiidae}

Farrodes yungaensis Dominguez \& Savage, 1987, E.R.Silva det.

Ocorrência: Terra Boa

Massartela fruhstorfferi Ulmer, 1943, E.R. Silva det.

Ocorrência: Lancinha

\section{Tricorythidae}

Tricorythodes sp., E.R. Silva det.

Ocorrência: Lancinha

\section{Caenidae}

Caenis sp., E.R. Silva det.

Ocorrência: Lancinha

Observações: Foram registrados seis gêneros em três grutas (das nove que possuem curso d'água), nunca tendo sido observados mais de três indivíduos. No trecho entre as duas cachoeiras da Gr. da Lancinha, a mais de $100 \mathrm{~m}$ do sumidouro, foram coletados ninfa e subimago de Massartela fruhstorfferi $\mathrm{e}$ subimagos ou imagos de Caenis sp., de Tricorythodes sp., de Farrodes yungaensis e de Callibaetis sp. Na Gr. Olhos d'Água encontrou-se um subimago de Pseudocleon sp.

\section{Ordem BLATTODEA}

\section{Blaberidae}

Blaberinae

? Petasodes sp., C.J. Becker det.

Ocorrência: Lancinha

\section{Epilampridae}

Epilamprinae

? Hedaia sp., C.J. Becker det.

Ocorrência: Toquinhas

Observações: Os dois registros de Blattodea nas cavernas paranaenses foram feitos com base em uma ninfa cada, e devido a isto não foi possível obter-se uma determinação precisa. Estas ninfas foram encontradas em acúmulos de folhas próximos à comunicações com o meio epígeo. Segundo TRAJANO (1990), os Blattodea são raramentes observados nas cavernas do Vale do Ribeira sendo mais frequentes nas cavernas situadas ao norte desta Província. 


\section{Ordem ENSIFERA}

\section{Phalangopsidae}

Strinatia sp., F.G.Mello det.

Ocorrência: Água Boa, Bacaetava, Bromados, Itacolombo, Jesuítas, Lancinha, Olhos d'Água, Pinheirinho, Pinheiro Seco, Terra Boa, Toca, Toquinhas

Observações: A espécie de grilo encontrada em todas as grutas visitadas é um trogloxeno. Adultos e principalmente ninfas sempre foram observados próximos à entradas. Alimentam-se provavelmente de detritos vegetais no exterior da gruta. Outras espécies do gênero Strinatia são encontradas em grutas areníticas e carbonáticas de São Paulo.

\section{Ordem PSOCOPTERA}

Ocorrência: Itacolombo, Toca

Observações: Os psocópteros alimentam-se de fungos e detritos vegetais. Os exemplares observados não apresentavam asas desenvolvidas e foram coletados em guano de hematófagos (Gr. de Itacolombo) e em armadilha de formalina iscada com fígado de galinha (Gr. da Toca).

\section{Ordem THYSANOPTERA}

\section{Ocorrência: Terra Boa}

Observações: Foi encontrado apenas um exemplar em guano de insetívoros a 200m da entrada na Gr. de Terra Boa. GNASPINI-NETTO (1989) também observou Thysanoptera em guano de insetívoros na caverna Refúgio do Marajoara (arenito, Amazonas).

\section{Ordem HETEROPTERA}

\section{Reduviidae}

Zelurus travassosi (Costa-Lima, 1940)

Ocorrência: Água Boa, Bacaetava, Bromados, Jesuítas, Itacolombo, Lancinha, Olhos d'Água, Pinheirinho, Pinheiro Seco, Terra Boa, Toca, Toquinhas

Observações: Os heterópteros reduviídeos são predadores encontrados em várias cavernas nas Américas. A espécie registrada, Zelurus travassosi, também ocorre em cavernas de São Paulo (TRAJANO, 1987) e de Minas Gerais (CHAIMOWICZ, 1986). No Paraná foi registrada em todas as grutas amostradas, com ocorrência de ninfas em todos os substratos de todas as regiōes das cavernas e de adultos sempre próximos a comunicações com exterior. Esta espécie foi observada predando os opiliões Goniosoma aff. badium (jovem, Gr. da Toca) e Daguerreia inermis (adultos, Gr. de Água Boa, Gr. dos Jesuítas e Gr. 
da Lancinha) e, segundo TRAJANO \& GNASPINI-NETTO (1990) são também predadores de grilos.

\section{Ordem HOMOPTERA}

Cixiidae, A. Sakakibara det.

Ocorrência: Jesuítas, Lancinha, Olhos d'Água, Pinheirinho, Terra Boa, Toquinhas

Observações: Os cixiídeos cavernícolas, tanto ninfas como adultos, alimentam-se sugando raízes que penetram na rocha e chegam até as cavernas (HOCH \& HOWARTH, 1989). Nas grutas do Paraná foram observados grupos de até 10 adultos, perto de raízes próximas de entradas (Gr. dos Jesuítas, Gr. Olhos d'Água, Gr. de Pinheirinho e Gr. de Toquinhas), ou em locais afastados, a mais de $200 \mathrm{~m}$ das entradas conhecidas (Gr. da Lancinha e Gr. de Terra Boa). Não foram encontradas ninfas.

\section{Ordem COLEOPTERA}

\section{Carabidae}

Carabinae

Tribo Bembidiini, S.A.Vanin det.

Ocorrência: Jesuítas

Paratachys sp., S.A. Vanin det.

Ocorrência: Jesuítas

Tribo Pterostichini, S.A.Vanin det.

Ocorrência: Lancinha

Tribo Scaritini

Ocorrência: Jesuítas

Histeridae, Gnaspini-Netto det.

Ocorrência: Água Boa

Hidrophilidae, Gnaspini-Netto det.

Ocorrência: Água Boa

\section{Cholevidae}

Adelopsis sp., Gnaspini-Netto det.

Ocorrência: Lancinha

Dissochaetus murrayi Reitter, 1884, Gnaspini-Netto det.

Ocorrência: Água Boa

Dissochaetus villosus Szymczakowski, 1961, Gnaspini-Netto det.

Ocorrência: Água Boa, Lancinha, Olhos d'Água, Pinheirinho, Toquinhas Dissochaetu vanini Gnaspini, 1991, Gnaspini-Netto det.

Ocorrência: Bacaetava, Itacolombo, Jesuítas, Lancinha, Olhos d'Água, Pinherinho, Pinheiro Seco, Terra Boa, Toca, Toquinhas 
Staphylinidae, Gnaspini-Netto det.

Ocorrência: Água Boa, Jesuítas, Lancinha

Ptilodactylidae, Gnaspini-Netto det.

Ocorrência: Jesuítas, Terra Boa

\section{Psephalidae}

Goniacerinae

cf. Sillilicus sp., Gnaspini-Netto det.

Ocorrência: Lancinha

cf. Strombopsis sp., Gnaspini-Netto det.

Ocorrência: Itacolombo, Lancinha

cf. Xybaris sp., Gnaspini-Netto det.

Ocorrência: Lancinha

\section{Elmidae}

Phanocerus sp., Gnaspini-Netto det.

Ocorrência: Lancinha

Anthribidae, Gnaspini-Netto det.

Ocorrência: Itacolombo, Lancinha, Pinheirinho

\section{Tenebrionidae}

Goniadera sp., S.A.Vanin det.

Ocorrência: Jesuítas

Observações: Os besouros estão representados nas cavernas estudadas por 10 famílias e pelo menos 18 espécies. Os carabídeos são predadores e foram encontrados em acúmulos de restos vegetais na Gruta dos Jesuítas e na margem do rio na Gr. da Lancinha. As espécies de Histeridae e Hidrophilidae, ambas predadores, foram encontradas em guano de hematófagos na Gr. da Água Boa. Da família Cholevidae registrou-se uma espécie de Adelopsis, uma única vez, e três de Dissochaetus, ambas guanófagas. D. murrayi, D.villosus e D. vanini são muito comuns nas cavernas do Mato Grosso do Sul e estão largamente distribuídas nas cavernas de São Paulo (GNASPINI, 1991). No Paraná foram encontradas populações de mais de 30 indivíduos, tanto larvas como adultos, principalmente em guano de hematófagos, mas também em guano de carnívoros. Exemplares de Dissochaetus spp. também foram coletados em armadilhas de formalina iscadas com fígado de galinha. Os estafilinídeos que podem ser predadores ou detritívoros, foram registrados em guano de hematófagos (Gr. de Água Boa) e no solo (Gr. da Lancinha). As espécies de Ptilodactlyidae alimentam-se de detritos vegetais e foram registradas larvas em tronco em decomposição (Gr. Jesuítas) e um adulto próximo a curso d'água. Alguns indivíduos de Pselaphidae foram encontrados em guano velho de hematófagos e provavelmente alimentam-se de ácaros. Um exemplar adulto de Phanocerus foi registrado na Gr. da Lancinha em curso d'água. Vários exemplares de Anthribidae, micofágos, foram encontrados em fezes de lontra, folhiço e no solo. Este é o primeiro registro desta família em cavernas no Brasil. As espécies de 
Dissochaetus, Staphylinidae, Pselaphidae e Anthribidae são provavelmente troglófilas, pois foram registradas populações de tamanho considerável, em todas as regiões da caverna, e apresentando evidências de reprodução no ambiente cavernícola em alguns casos. Nada se pode afirmar quanto aos demais besouros, visto que apenas um exemplar foi registrado em cada caverna.

\section{Ordem TRICHOPTERA}

\section{Hydropsychidae}

Smicridea sp., L. Oliveira det.

Ocorrência: Lancinha, Olhos d'Água, Terra Boa, Toquinhas

\section{Philopotamidae}

Chimarra sp., L. Oliveira det.

Ocorrência: Terra Boa

Observações: Os Tricópteros são insetos crepusculares e noturnos e foram encontrados em muitas cavernas do Brasil (TRAJANO, 1987). Nas cavernas paranaenses, Chimarra sp. foi registrada apenas uma vez próxima a entrada. Smicridea sp. mostrou-se bastante comum, tendo sido observado até 10 adultos voando próximos ao rio, em locais distantes das comunicaçōes com o exterior. As populações deste gênero de tricóptero parecem ser troglófilas, situação igualmente observada no Alto Ribeira (TRAJANO comunicação pessoal).

\section{Ordem LEPIDOPTERA}

\section{Noctuidae}

Ophiderinae

Latebraria amphipyroides Guenée, 1852, V. Becker det.

Ocorrência: Água Boa, Bromados, Jesuítas, Lancinha, Olhos d'Água, Pinheirinho, Terra Boa, Toca, Toquinhas

Tineidae, V. Becker det.

Ocorrência: Água Boa, Itacolombo, Jesuítas, Toquinhas

Observações: As mariposas Noctuidae foram observadas pousadas na parede em locais onde a luz solar não incidia diretamente e nunca a mais de $20 \mathrm{~m}$ da entrada, tratando-se de trogloxenos. Os Tineidae apresentam um largo espectro de preferências alimentares, desde plantas até guano ou animais mortos e são encontrados em cavernas de várias regiões do mundo (ROBINSON, 1980). No Paraná foram encontrados em fezes de lontra (larvas e adultos, Gr. de Itacolombo, Gr. dos Jesuítas) e em guano de hematófagos (larvas, Gr. de Água Boa, Gr. da Lancinha), em todas as ocasiões próximos a entradas. GNASPINI-NETTO (1989) registrou larvas de Tineidae em guano de hematófagos, frugívoros e carnívoros em cavernas de São Paulo e Mato Grosso. 


\section{Ordem DIPTERA}

Tipulidae, S.R. Malkowski det.

Ocorrência: Olhos d'Água

\section{Psychodidae}

Pericoma sp., E.A.B. Galati det.

Ocorrência: Itacolombo, Lancinha, Pinheirinho

Telmatoscopus sp., E.A.B. Galati det.

Ocorrência: Lancinha

Trichonyia sp., E.A.B. Galati det.

Ocorrência: Itacolombo, Lancinha

\section{Culicidae}

Ocorrência: Água Boa, Itacolombo, Lancinha, Olhos d'Água, Toca, Toquinhas

Simuliidae, C.J.B. Carvalho det.

Ocorrência: Lancinha

Ceratopogonidae, D.S. Amorim det.

Ocorrência: Lancinha, Terra Boa

\section{Chironomidae}

Orthocladiinae, S. Trivinho-Strixino det.

Ocorrência: Lancinha

\section{Anisopodidae}

Sylvicola annuliferus (Edwards, 1923), D.S. Amorin det.

Ocorrência: Lancinha

\section{Mycetophilidae}

Leia tricinctiventris Enderlein, 1910, D.S. Amorin det.

Ocorrência: Lancinha

\section{Keroplatidae}

Neoplatyra sp., D.S. Amorin det.

Ocorrência: Terra Boa

\section{Sciaridae}

gênero indet.

Ocorrência: Toca

Bradysia sp., D.S. Amorin det.

Ocorrência: Pinheirinho

Ctenosciara sp., D.S. Amorin det.

Ocorrência: Lancinha, Terra Boa, Toquinhas

Odontosciara sp., D.S. Amorin det.

Ocorrência: Toquinhas

Pnyxia sp., D.S. Amorin det. 
Ocorrência: Pinheirinho

Pseudosciara sp., D.S. Amorin det.

Ocorrência: Pinheirinho

Cecidomyiidae, D.S. Amorin det.

Ocorrência: Lancinha

\section{Milichiidae}

Pholeomyia, sp. A.L. Serra det.

Ocorrência: Água Boa

\section{Drosophilidae}

Drosophila melanogaster, Meigen, 1830 C.R. Vilela det.

Ocorrência: Água Boa

\section{Fanniidae}

Fannia albitarsis Stein, 1911, S.R. Malkowski det.

Ocorrência: Água Boa, Itacolombo, Lancinha, Olhos d'Água, Terra Boa

\section{Muscidae}

Psilochaeta pampeana (Shannon \& Del Ponte, 1926), C.J.B. Carvalho det.

Ocorrência: Água Boa, Bacaetava, Itacolombo, Jesuítas, Lancinha, Olhos d'Água, Pinheiro Seco, Toca

Phoridae, A.P. Prado det.

Ocorrência: Água Boa, Bacaetava, Bromados, Itacolombo, Jesuítas, Lancinha, Olhos d'Água, Pinheirinho, Terra Boa, Toca, Toquinhas

Observações: Os dípteros encontrados pertencem a 16 famílias, normalmente assinaladas no ambiente cavernícola, segundo MATILE (1970); dentre estas, Psychodidae, Milichiidae, Fanniidae, Muscidae, Drosophilidae e Phoridae são guanófilas. Larvas de Fannia albitarsis foram encontradas em guano fresco de hematófagos. Larvas de Psilochaeta pampeana foram observadas em guano de hematófagos e carnívoros, formando agregados de mais de 30 indivíduos, adultos foram vistos ovipondo em guano de hematófagos e carnívoros na Gr. Toquinhas. Ambas as espécies sempre foram encontradas em acúmulos de guano próximos a comunicações com o exterior. Portanto, estas populações são consideradas trogloxenas. Larvas de Pholeomyia sp. foram registradas em guano de hematófagos e larvas de Psychodidae, em guano de carnívoros. Os adultos de Culicidae e de Sciaridae foram observados nas paredes das entradas, sendo notadamente trogloxenos. Os Chironomidae foram registrados uma única vez na Gr. da Lancinha, próximo a rio. Dípteros Simuliidae foram encontrados próximos a ressurgência, uma única vez, na Gr. da Lancinha, sendo considerados acidentais. Os dípteros mais comuns são os Phoridae, troglófilos, cujas larvas e adultos foram encontrados em guano de hematófagos em todas as zonas da caverna. Estes dípteros foram capturados em armadilha iscada com fígado de galinha, na Gr. da Lancinha e Gr. da Toca. Adultos de Drosphila melanogaster foram obtidos a partir, da criação em labo- 
ratório, de larvas encontradas em guano de hematófagos situado próximo à entrada da Gr. Água Boa. Este registro é de notável interesse pois até o presente a única espécie de Drosophila registrada em cavernas brasileiras (São Paulo, Mato Grosso do Sul, Goiás, Minas Gerais e Pará) era D. eleonorae (TOSI et al., 1990). Cabe destacar que as larvas de D. melanogaster vivem normalmente em frutas, legumes e flores podres, em cogumelos (CARSON, 1965) e este parece ser o primeiro registro desta espécie cosmopolita em guano de morcegos (C.R. Vilela, com.pes).

\section{Ordem HYMENOPTERA}

\section{Braconidae}

Alysiinae

Chorebus sp., H. Fischer \& V. Graf det.

Ocorrência: Água Boa, Jesuítas, Lancinha, Toca, Pinheiro Seco.

Observações: Estes microhimenópteros foram encontrados várias vezes próximos a guano de hematófagos e em várias regiões nas cavernas. Chorebus sp. provavelmente é parasitóide de ovos ou larvas de dípteros. Os exemplares coligidos nas cavernas paranaenses constituem o primeiro registro do gênero para o Brasil (H. Fischer com. pes.).

\section{DISCUSSÃO}

\section{Distribuição.}

A fauna de invertebrados das cavernas carbonáticas do sul Vale do Ribeira, de uma forma geral é semelhante à de outras cavernas brasileiras, sendo bastante similar à das cavernas carbonáticas de São Paulo. Fato já esperado, visto que ambas estão situadas na Prov. Esp. do Vale do Ribeira que apresenta características ambientais, de uma forma geral, semelhantes. Diferenças conspícuas devem-se principalmente à ausência nas grutas paranaenses de certos táxons que são relativamente comuns nas cavernas carbonáticas de São Paulo. Este é o caso dos isópodos terrestres Oniscidae e Styloniscidae; diplópodos Cryptodesmidae; e Chelodesmidae; opiliôes Tricommatidae; tricópteros Calamoceratidae; e dípteros Keroplatidae do gênero Neoditomyia. Notável é a ausência dos grilos troglófilos do gênero Endecous, que habitam a zona afótica de muitas cavernas de São Paulo, e normalmente quando estes estão ausentes, Strinatia spp. geralmente ocupam toda a caverna (TRAJANO \& GNASPININETTO, 1991a). Nas cavernas estudadas, nas quais Endecous sp. não ocorre, Strinatia sp. não colonizou a zona afótica.

Alguns grupos comuns e amplamente distribuídos na parte norte do Vale do Ribeira foram observados raras vezes nas grutas paranaenses. Este é o caso dos coleópteros Cholevidae do gênero Adelopsis, Ptilodactylidae e Elmidae; e dos dípteros Ceratopogonidae, Chironomidae e Simuliidae. A mariposa Latebraria amphipyroides foi encontrada em quase todas as grutas estudadas, 
porém nas de São Paulo, espécimes de Noctuidae não são tão comuns.

O registro de apenas dois grupos de organismos aquáticos, decápodos Aegla e náiades de Ephemeroptera, não significa necessariamente que a fauna desse ambiente é pouco diversificada. Reflete, antes, o pouco esforço dispendido na coleta neste ambiente. Cabe destacar que foi descrita a subespécie de copépodo Elaphoidella bidens subterranea Nogueira, 1959, da Gr. de Itaperussu $\left(25^{\circ} 13^{\prime} 43^{\prime \prime} \mathrm{S}, 4^{\circ} 20^{\prime} 20^{\prime \prime} \mathrm{W}\right)$, situada no Paraná, não visitada no presente estudo.

\section{Estratificação e zonação na caverna.}

As cavernas de grandes dimensões e com locais distantes de comunicações com o exterior podem ser divididas em quatro zonas, segundo suas características ambientais: zona de entrada, zona de penumbra, zona de transição e zona profunda (HOWARTH, 1983). Para as cavernas brasileiras, no entanto, não existem estudos disponíveis sobre o topoclima subterrâneo, e estas quatro zonas não podem ser identificadas com facilidade, sendo possível apenas distinguir uma fauna da entrada e proximidades (zona de entrada + zona de penumbra) e uma fauna da região afótica (zona de transição + zona profunda), TRAJANO (1987).

A região da entrada e proximidades apresenta uma fauna associada às paredes e teto (parietal) e outra associada a recursos alimentares. Nas grutas estudadas, as principais fontes de alimento, observadas nesta região, são os detritos vegetais e guano de hematófagos e carnívoros, onde são encontrados principalmente trogloxenos e acidentais.

Os detritos vegetais normalmente entram neste ambiente carreados pelo vento e pelo rio formando acúmulos onde se pode observar pseudoescorpiōes, os ácaros de que se alimentam (possivelmente Steganacarus fonseciai, Tectocepheus americanus, Quadrioppia sp. e Pergalumna nasica) e isópodos (Trichorhina sp. e Philosciidae). É interessante notar que as larvas dos dípteros Psilochaeta pampeana e Fannia albitarsis foram observadas em guano de hematófagos apenas na zona de entrada. Larvas de $P$. pampeana também foram encontradas em guano de carnívoros e os adultos observados entrando e saindo da caverna, tratando-se portanto de um guanobio trogloxeno. Estas observações diferem das de GNASPINI (1992) que considerou esta espécie como trogloxena e restrita ao guano de hematófagos. Insetos e aracnídeos são os principais grupos que compõem a associação parietal nas entradas e proximidades e, provavelmente, estão neste local devido a fatores como temperatura mais amena, umidade do ar maior e disponibilidade de alimento (JEFFERSON, 1983). As aranhas errantes, como Enoploctenus sp., e sedentárias, como Blechroscelis sp., são características deste local, sendo que a última foi observada carregando ootecas diversas vezes. Outro aracnídeo que reproduz na zona de entrada é o opilião Goniosoma aff. badium, que pode formar agregados de até 34 indivíduos, e eventualmente alimenta-se neste local. Dípteros como Culicidae, Sciaridae e a mariposa Latebraria amphipyroides permanecem durante o dia na região de entrada, saindo durante ou após o entardecer. Também 
é característico desta região o grilo Strinatia sp. É interessante citar que em cavernas com grande disponibilidade de alimento, como as da Prov. Esp. Arenitica de Altamira-Itaituba, grupos que normalmente ocorrem na entrada de cavernas "pobres" em alimento, podem ocupar zonas mais profundas (TRAJANO \& MOREIRA, 1991).

Em cursos d'água e em suas galerias encontram-se, na região afótica, os decápodos Aegla paulensis, efemerópteros, tricópteros Smicridea sp., aranhas Plato sp. e opiliões Daguerreia inermis. Estes dois últimos apresentam populações maiores, neste local, que em galerias secas e distantes do rio. A explicação para este fato talvez esteja ligada à maior quantidade de energia disponível a estas espécies nos locais com cursos d'água. Nas galerias secas, onde a influência do rio não se faz sentir de forma direta, observa-se uma fauna normalmente associada a depósitos de guano, composta por ácaros (e.g. Macrocheles muscaedomesticae, Proctolaelaps sp.), diplópodos (Pseudonannolene strinatï, Katantodesmus sp.), colêmbolos (e.g. Acherontides sp. e Paronella sp.), coleópteros (Cholevidae e Pselaphidae) e dípteros (Phoridae). A maioria dos representantes da fauna da zona afótica podem ser encontrados na região de entrada e proximidades, à exceção de animais que apresentam especializações relacionadas à vida cavernícola (TRAJANO, 1987), como é o caso de Katantodesmus sp. e talvez Acherontides sp.

Animais que comumente ocorrem tanto em locais de entrada quanto em zonas afóticas são os aracnídeos Ctenus fasciatus, e os insetos das famílias Paronellidae, Reduviidae, Cixiidae, Cholevidae, Anthribidae, Hydropsychidae, Tineidae, Phoridae e Braconidae.

\section{Classificação ecológica dos cavernícolas.}

Os cavernícolas são classificados segundo o seu grau de especialização e dependência em relação aos meios hipógeo e epígeo, em três categorias: troglóbios, troglófilos e trogloxenos. Os animais considerados acidentais não apresentam uma relação estreita com este ambiente e portanto não podem ser considerados cavernícolas verdadeiros. Para enquadrar os cavernícolas nesta classificação, adotou-se os critérios de TRAJANO (1987), baseados principalmente na morfologia, mobilidade e hábitos alimentares, uma vez que o conhecimento sobre ecologia e distribuição das espécies neotropicais é bastante restrito. Devido a isto, alguns troglóbios podem ter sido aqui classificados como troglófilos.

Os dois únicos organismos que apresentam troglomorfismos e parecem ser troglóbios são o diplópode Katantodesmus sp. e o colêmbolo Acherontides sp., ambos associados a guano de hematófagos.

A maioria dos cavernícolas do Paraná são troglófilos, pois formam populações que efetivamente se alimentam e reproduzem nas cavernas, e apresentam morfologia semelhante às populações epígeas e podem ser encontrados a distâncias consideráveis das comunicações com o exterior. Os troglófilos mais notáveis são os pseudoescorpiōes Chernetidae; as aranhas 
Ctenus fasciatus, Loxosceles gaucho e Plato sp.; os diplópodos Pseudonannolene strinatii Strongylosomidae; colêmbolos (e.g. Paronellidae e Isotomidae); os coleópteros Dissochaetus spp. Pselaphidae e Anthribidae; tricópteros Smicridea sp.; os dípteros Phoridae e os microhimenópteros parasitóides Chorebus sp.

Algumas espécies de ácaros apresentam uma distribuição relativamente ampla nas cavernas estudadas, utilizando diversos recursos alimentares. Em algumas grutas foram observados mais de 10 indivíduos. Estas características, aliadas a um ciclo de vida curto e aparente ausência de especialização à vida no ambiente hipógeo, parecem conferir o "status" de troglófilo aos seguintes táxons: Macrocheles muscaedomesticae, Proctolaelaps sp., Spinibdella cronini, Galumna inexa e Peloribates anomalus. Também podem ser incluídos nesta categoria Tyrophagus putrescentiae e Blattissocius tarsalis, caso não seja comprovada a infestação nos funis por estes ácaros, oriundos da poeira.

Os trogloxenos normalmente utilizam-se das zonas de entrada como abrigo e/ou para reprodução, podendo alimentar-se nestes locais. Este é o caso das aranhas Enoploctenus sp., Trechalea keyserlingi, Achaearanea sp., do opilião Goniosoma aff. badium, do grilo Strinatia sp., da mariposa Latebraria amphipyroides e dos dípteros Psychodidae, Scaridae, Culicidae, Fannia albitarsis e Psilochaeta pampeana. A aranha Blechroscelis sp. alimenta-se e reproduz-se na zona de entrada, contudo não forma populações numerosas, sendo também considerada trogloxena.

Para vários grupos, comumente encontrados nas cavernas brasileiras, não foi possível precisar se eram troglófilos, trogloxenos ou acidentais, devido à escassez de registros. Os principais grupos que permanecem duvidosos são a maioria dos ácaros, efeméridas, psocópteros, cixiídeos, coleópteros das famílias Histeridae, Hidrophilidae, Cholevidae (Adelopsis sp.), Ptilodactylidae, Elmidae e os dípteros Psychodidae, Chironomidae e Milichiidae.

AGRADECIMENTOS. A Dra. Eleonora Trajano e M.Sc. Pedro Gnaspini-Netto pelo apoio, críticas e sugestões em todas as fases de desenvolvimento do presente estudo. A todos os especialistas, cujos créditos foram dados no texto, pela identificação do material coligido e pelos valiosos comentários. A Maria Martha Argel de Oliveira pela leitura crítica do manuscrito. Aos amigos espeleólogos do Grupo de Estudos Espeleológicos Açungui, de Curitiba, em especial a Darci Paulo Zakrzewski, pelo auxílio durante as coletas nas cavernas e pelas informações fornecidas. Este estudo foi parcialmente financiado pelo CNPq (processos $\mathrm{n}=803.013-87.6$ e 804.495-88.2).

\section{REFERÊNCIAS BIBLIOGRÁFICAS}

AB'SABER, A.N. 1977. Os domínios morfoclimáticos na América do Sul. Primeira aproximação. Geomorfologia 52: 1-21.

BARR,, T.C. \& J.R. HOLSINGER, 1985. Speciation in cave faunas. Ann. Rev. Ecol. Syst. 16: 313-337.

CARSON, H. 1965. Chromosomal morphism in geographically widespread species of Drosophila, p. 503-531. In: BAKER, H.G. \& G.L. STEBBINGS (eds). The Genetics of colonizing species. New York, Academic Press. 
CARVAlHO, C.J.B. 1989. Revisão de Psilochaeta Stein e descrição de Dalcyella gen.n. do Chile (Diptera, Muscidae). Revta bras. Zool. 6 (3): 485-506.

CHAIMOWICZ, F. 1984. Levantamento bioespeológico de algumas grutas de Minas Gerais. Espeolo-Tema (Sociedade Brasileira de Espeologia) 14: 97-107.

-1986. Observações preliminares sobre o ecossistema da gruta Olhos d'Água, Itacarambi, MG. Espeleo-Tema (Sociedade Brasileira de Espeleologia) 15: 65-77.

CODDINGTON, J.A. 1986. The genera of the spider family Theridiosomatidae. Smithsonian Contr. Zool. 422: 1-96.

CULVER, D.C. 1982. Cave life: Evolution and Ecology. Cambridge, Harvard Univ. Press., 189p.

DESSEN, E.M.B.; V.R. ESTON;M.T. TEMPERINI-BECK \& E. TRAJANO. 1980. Levantamento preliminar da fauna de cavernas de algumas regiões do Brasil. Ciênc. \& Cult. 32 (6): 714-725.

EICKSTEDT, V.R.D. 1975. Aranhas coletadas nas grutas calcárias de Iporanga, São Paulo, Brasil. Mem. Inst. Butantan 39: 61-71.

FLECHTMANN, C.H.W. 1977. Ácaros de importância médico-veterinária. São Paulo, Nobel, $2^{a}$ ed., 192p.

. 1983. Ácaros de importância agrícola. São Paulo, Nobel, $5^{a}$ ed., 189p.

GNASPINI-NETTO, P. 1989. Análise comparativa da fauna associada a depósitos de guano de morcegos cavernícolas no Brasil. Primeira aproximação. Revta bras. Ent. 33 (2): 183-192.

GNASPINI, P. 1991. Brazilian Cholevidae (Coleoptera), with emphasis on cavernicolous species. I. Genus Dissochaetus. G. it. Ent. 5: 325-340.

. 1992. Bat guano ecosystems a new classfication and some considerations with special references to neotropical data. Mem. Biospeol. 19: $135-138$.

GODOY, N.M. 1986. Nota sobre a fauna cavernícola de Bonito, MS. Espeleo-Tema (Sociedade Brasileira de Espeleologia) 15: 79-90.

HOCH \& HOWARTH, 1989. The evolution of cave-adapted cixiid planthoppers in volcanica and limestone caves in north Queesland, Australia (Homoptera: Fulgoroidea). Mem. Biospeol. 16: 17-24.

HOWARTH, F.G. 1983. Ecology of cave arthropods. Ann. Rev. Ent. 28: 365-389.

JEFFERSON, G.T. 1983. The threshold fauna. Studies Speleol. 4: 53-59.

KARMANN, I. \& L.E. SÁNCHEZ, 1986. Speleological provinces in Brazil. Res. Congresso Internacional de Espeleologia, Barcelona, p.151-153.

KLEIN, R.M. 1962. Fitofisionomia e notas sobre a vegetação para acompanhar a planta fitoggeográfica de partes dos municípios de Rio Branco do Sul Bocaiúva do Sul Almirante Tamandaré e Colombo (PR). Bol. Univ. Fed. Paraná Geografia Física 3: 1-33.

KRANTZ, G.W. 1975. A manual of Acarology. Oregon State University Book 
Stores, $2^{\mathrm{a}}$ ed., $335 \mathrm{p}$.

MAACK, R. 1981. Geografia física do Estado do Paraná. Rio de Janeiro, Livraria José Olympio, 450p.

MATILE, L. 1970. Les dipteres cavernicoles. Ann. Speleol. 25 (1): 179-222.

NOGUEIRA, M.H. 1959. O gênero Elaphoidella (Harpacticoidea-Cop. Crust.) nas águas do Paraná. Dusenia 8 (2): 61-68.

PINTO-DA-ROCHA, R. 1989. Levantamento preliminar da fauna de invertebrados das cavernas calcárias do Estado do Paraná. Res. 20º Congresso Nacional de Espeleologia, Brasilia, p.9-13.

ROBINSON, G.S. 1980. Cave-dwelling tineid moths: a taxonomic review of the world species. Trans. British Cave Res. Assoc. 7 (2): 823-120.

SCHUBART, O. 1953. Sobre os diplópodos dos Estados do Paraná e Santa Catarina. Arq. Mus. Paranaense 10 (3): 77-140.

SOARES, B.A.M. \& H.E.M. SOARES. 1947. Opiliões pertencentes a coleção Gert Hatschbach (Opiliones Gonyleptidae, Phalangodidae, Phalangiidae). Papéis Dep. Zool., São Paulo, 8 (18): 209-230.

SOCIEDADE BRASILEIRA DE ESPELEOLOGIA. 1989. Cadastro Nacional de Cavidades Naturais. Índice de dados sobre as cavernas do Brasil. São Paulo, 222p.

TOSI, D.; M. MARTINS; C.R. VILELA \& M.A.Q.R. PEREIRA. 1990. On a new cave-dwelling species of bat-guano-breeding Drosophila closely related to D. repleta Wollaston (Diptera, Drosophilidae). Revta bras. Genet. 13 (1): 19-31.

TRAJANO, E. 1985. Ecologia de populações de morcegos cavernícolas em uma região cárstica do sudeste do Brasil. Revta bras. Zool. 2 (5): 255-320.

— 1987. Fauna cavernícola brasileira: composição e caracterização preliminar. Revta bras. Zool. 3 (8): 533-561.

TRAJANO, E. \& GNASPINI-NETTO. 1991a. Composição da fauna cavernícola brasileira, com uma análise da distribuição dos táxons. Revta bras. Zool. 7(3): 383-407.

. 1991b. Notes on the food webs in caves of Southeastern Brazil. Mem. Biospeol. 18: 75-79.

Recebido em 25.04.1991; aceito em 25.XI.1993. 